\title{
REGULAR COMPACTIFICATIONS OF CONVERGENCE SPACES
}

\author{
G. D. RICHARDSON AND D. C. KENT
}

Abstract. This note gives a simple characterization for the class of convergence spaces for which regular compactifications exist and shows that each such convergence space has a largest regular compactification.

Introduction. It has been shown by Wyler [5] that for every Hausdorff convergence space $S$ there is a regular (including Hausdorff) compact convergence space $S^{*}$ and a continuous map $j: S \rightarrow S^{*}$ with the following property: for every continuous map $f: S \rightarrow T$, where $T$ is regular and compact, there is a unique continuous map $g: S^{*} \rightarrow T$ such that $f=g \circ j$. Richardson [4] obtained a similar result, but with the following important distinctions: (1) the compactification space $S^{*}$ is Hausdorff but not necessarily regular (for convergence spaces, Hausdorff plus compact does not imply regular); (2) the map $j$ is a dense embedding. But there is in general no largest Hausdorff compactification, and indeed the number of distinct maximal Hausdorff compactifications can be quite large.

The conclusions of both [4] and [5] suggest that a more satisfactory compactification theory for convergence spaces might result from an investigation of regular compactifications, although it is known (see [2]) that there are regular convergence spaces which cannot be embedded in any compact regular space. What we obtain in this note is a characterization of the class of convergence spaces for which regular compactifications exist, and we show that each such convergence space has a largest regular compactification.

1. For basic information about convergence spaces the reader is asked to refer to [1] and [2]. If there is no possibility of confusion, a convergence space $(S, q)$ will be denoted simply by $S$. A space is regular if it is Hausdorff and has the property: $\mathscr{F}$ converges to $x$ implies that the closure of $\mathscr{F}$ (denoted $\mathrm{cl} \mathscr{F}$ ) converges to $x$. We shall denote by $\operatorname{cl}_{S} A$ the closure of a subset $A$ of a convergence space $S$. The pretopological modification $\pi S$ of a convergence space $(S, q)$ is the space $(S, p)$, where $p$ is the finest pretopology

Received by the editors March 3, 1971.

AMS 1969 subject classifications. Primary 5410, 5453, 5423; Secondary 5422.

Key words and phrases. Regular convergence spaces, regular compactification of convergence spaces.

(c) American Mathematical Society 1972 
on $S$ coarser than $q$; the topological modification $\lambda S$ is defined analogously. Recall that $\pi S=\lambda S$ iff the closure operator $\mathrm{cl}_{S}$ is idempotent.

If $A$ is a subset of a convergence space $S$, then the subspace defined by $A$ (also denoted by $A$ ) is given as follows: A filter $\mathscr{F}$ on $A A$-converges to $x$ in $A$ iff the filter on $S$ generated by $\mathscr{F} S$-converges to $x$.

Proposition 1. The closure operator for a compact regular convergence space is idempotent.

Proof. Let $S$ be compact and regular, $A$ a subset of $S$. By the theorem of [4], the identity map from $A$ into $S$ has a continuous extension $f$ to $A^{*}$, the Stone-Čech compactification of $A$. Since $f\left(\mathrm{cl}_{A^{*}} A\right)=f\left(A^{*}\right) \subseteq \mathrm{cl}_{S} A$ by continuity of $f$ and $f\left(A^{*}\right)$ is compact and hence $S$-closed, it follows that $\operatorname{cl}_{S} A$ is closed.

Proposition 2. If $A$ is a subspace of $S$, then $\pi A$ is the subspace of $\pi S$ determined by $A$.

Proposition 3. If $A$ is a subspace of a compact regular convergence space, then $\pi A$ is Hausdorff and topological.

Proof. Let $S$ be a compact regular convergence space containing $A$. By Proposition 2 it suffices to show that $\pi S$ is Hausdorff and topological; that it is topological follows from Proposition 1. To see that $\pi S$ is Hausdorff, let $\mathscr{F}$ be an ultrafilter in $\pi S$ which converges to both $x$ and $y$. By compactness $\mathscr{F}$ converges in $S$ to some point $z$ and regularity guarantees that $\mathrm{cl}_{S} \mathscr{F}$ also converges in $S$ to $z$. But each neighborhood of $x$ is in $\mathscr{F}$, so $x$ is in each member of $\operatorname{cl}_{S} \mathscr{F}$ and hence the Hausdorffness of $S$ implies that $x=z$. Similarly, $y=z$, and so $x=y$.

2. A compactification $(T, f)$ of a convergence space $S$ consists of a compact convergence space $T$ along with a dense embedding $f$ of $S$ into $T$. For a different definition, see $\S 7$ of [3].

If $(T, f)$ is a compactification of $S$, then it is a simple matter to verify that $(\pi T, f)$ is a compactification of $\pi S$. From this fact and Proposition 3 we deduce the next result.

Proposition 4. If $(T, f)$ is a regular compactification of $S$, then $(\pi T, f)$ is a Hausdorff (topological) compactification of $\pi S$.

THEOREM 1. A regular convergence space $S$ has a regular compactification iff $\pi S$ is a completely regular topological space and each ultrafilter which is finer than the neighborhood filter at $x S$-converges to $x$ for all $x$ in $S$.

Proof. Assume the given conditions. Then $\pi S$ has a topological compactification $(T, f)$. Let $T_{1}$ be the convergence space consisting of the set $T$ equipped with the finest convergence structure $r$ on $T$ which satisfies the following conditions: if $f(S)$ belongs to $\mathscr{G}$, then $\mathrm{cl}_{T} \mathscr{G} r$-converges to $x$ 
in $f(S)$ iff $f^{-1}(\mathscr{G}) S$-converges to $f^{-1}(x)$; if $\mathscr{F}$ is an ultrafilter such that $T$ $f(S)$ belongs to $\mathscr{F}$, then $\operatorname{cl}_{T}(\mathscr{F}) r$-converges to $x$ in $f(S)$ iff $\mathscr{F} T$-converges to $x$; if $\mathscr{H}$ is an ultrafilter on $T$, then $\mathrm{cl}_{T} \mathscr{H} r$-converges to $y$ in $T-f(S)$ iff $\mathscr{H} T$-converges to $y$.

By this construction, it is clear that $T_{1}$ and $T$ coincide relative to ultrafilter convergence, and so the closure operators for these spaces coincide. The fact that $T_{1}$ is regular can be established with the aid of the following lemma: If $\mathscr{F}$ is an ultrafilter on $T-f(S)$ which $T$-converges to $x$ in $f(S)$, then there is an ultrafilter $\mathscr{G}$ on $S$ which $S$-converges to $f^{-1}(x)$ such that $f^{-1}\left(\mathrm{cl}_{T} \mathscr{F}\right) \geqq \mathrm{cl}_{S} \mathscr{G}$. Finally, it is easy to establish that $f: S \rightarrow T_{1}$ is a dense embedding.

If $S$ has a regular compactification $(T, f)$, then $\pi S$ is a completely regular topological space by Proposition 3 . To show that $S$ has the second property, let $\mathscr{F}$ be an ultrafilter finer than the $S$-neighborhood filter at $x$. Let $y$ be the point in $T$ to which $f(\mathscr{F}) T$-converges. Since $f(\mathscr{F})$ is finer than the neighborhood filter for $f(x), f^{\prime}(x) \geqq \mathrm{cl}_{T} f(\mathscr{F})$, and so necessarily $y=f(x)$. Thus $f^{-1}(f(\mathscr{F}))=\mathscr{F} S$-converges to $x$ since $f$ is an embedding.

A regular compactification $T$ of a convergence space $S$ is a Stone- $\breve{C} e c h$ regular compactification if each continuous function from $S$ into a compact regular space has a continuous extension to $T$.

THEOREM 2. If a convergence space has a regular compactification, then it has a Stone-Čech regular compactification.

Proof. Let $S$ be a convergence space with a regular compactification; let $(T, f)$ be the (topological) Stone-Čech compactification of $\pi(S)$, and let $\left(T_{1}, f\right)$ be the regular compactification of $S$ constructed above. If $g$ is any continuous function from $S$ into a compact regular space $R$, then $g$ : $\pi S \rightarrow \pi R$ has a unique continuous extension $h: T \rightarrow \pi R$ such that $h \circ f=g$, and it follows easily that $h: T_{1} \rightarrow R$ is also continuous.

\section{REFERENCES}

1. D. C. Kent, Convergence quotient maps, Fund. Math. 65 (1969), 197-205. MR 40 \#3497.

2. D. C. Kent and G. D. Richardson, Minimal convergence spaces, Trans. Amer. Math. Soc. 160 (1971), 487-499.

3. J. F. Ramaley and O. Wyler, Cauchy spaces. II, Math. Ann. 187 (1970), 187-199.

4. G. D. Richardson, A Stone-Čech compactification for limit spaces, Proc. Amer. Math. Soc. 25 (1970), 403-404. MR 41 \#992.

5. O. Wyler, The Stone-Čech compactification for limit spaces, Notices Amer. Math. Soc. 15 (1968), 169. Abstract \#653-306.

Department of Mathematics, East Carolina University, Greenville, North Carolina 27834

Department of Mathematics, Washington State University, Pullman, WashINGTON 99163 